\title{
ROLL-GAP LUBRICATION EXCELLENCE TO OPTIMIZE HOT AND COLD ROLLING PROCESSES*
}

Konrad Krimpelstätter ${ }^{1}$ Christoph Pröll ${ }^{2}$

Martin Bergmann ${ }^{3}$

\begin{abstract}
The need for more effective and flexible roll-gap lubrication technologies in hot and cold rolling mills is steadily increasing due to ever-greater requirements placed on the rolling process and the final products. In particular, the rolling of high-strength steels poses enormous demands for both the rolling mill and the coolant/lubricant to be used. The presented paper focuses on two innovative technologies for highly efficient roll-gap lubrication for hot and cold rolling, which were developed utilizing extensive knowledge and expertise about the tribological interactions in the roll-gap. The advanced work-roll lubrication package (A-WRL) for hot rolling and ESP mills is using specially designed mixer-nozzle units to mix the water/oil emulsion very close to the nozzles to minimize the risk for contamination and clogging. The nozzles are quickly exchangeable and are optimized to ensure uniform lubricant distribution, high plateout efficiency and minimum oil consumption. This eco-friendly solution does not require any chemical additives or cleaning agents and significantly reduces electrical energy consumption and work-roll wear. The roll cooling system on mill entry and exit side remains switched-on, which is of vital importance especially for highperformance hot strip mills. Minimum Quantity Lubrication MQL® represents a new generation of roll-gap lubrication technologies for cold rolling, which applies minimum amounts of pure rolling oil finely atomized with pressurized air directly onto the work roll surface. This allows controlling the oil film thickness depending on actual process and product parameters, providing increased lubrication flexibility, significant improvement of strip surface cleanliness through reduction of strip wear. MQL ${ }^{\circledR}$ was successfully installed and tested on two industrial tandem cold rolling mills in Europe. Advanced WRL and MQL $®$ represent Primetals' latest technologies that feature a robust and reliable system comprising standardized interfaces and flexible communication options. Both packages can easily be integrated into existing or new hot strip mills, ESP lines or cold mills.
\end{abstract}

Keywords: Roll-gap lubrication; Work roll lubrication; WRL; A-WRL; Emulsion lubrication; Minimum quantity ILubrication, MQL; Plate-out.

1 DR, Head of Technology \& Innovation Hot and Cold Rolling, Primetals Technologies Austria $\mathrm{GmbH}$, Linz, Upper Austria, Austria

2 DI, Technology Hot and Cold Rolling, Primetals Technologies Austria GmbH, Linz, Upper Austria, Austria

3 DI DR, Technology Hot and Cold Rolling, Primetals Technolgies Austria GmbH, Linz, Upper Austria, Austria 


\section{INTRODUCTION}

The growing demands of steel producers for optimized processes to increase product quality, to reduce operational costs and to extend their product mix especially for rolling new steel grades and thinner and thicker gauges (pipe grades) on hot strip mills resp. thinner gauges on cold mills, requires new roll-gap lubrication solutions which are more efficient, less oil consuming and more flexible.

The rolling of high-strength steels poses enormous demands on rolling mills and coolants/lubricants. The applied medium must be optimized for all rolling applications, and it must be suited to meet the targeted quantity and quality requirements for various steel grades.

Based on these customer demands Primetals Technologies Austria is working since many years to extend the knowledge and expertise on lubrication and cooling to better understand the complex tribological phenomena (e.g. [1]) in the roll-gap and to support customers with leading-edge innovations.

A further important challenge is to design a robust lubrication system with high availability and minimum maintenance requirements. Primetals consequently puts effort to develop optimized technology packages for hot and cold rolling that are needed for operational success. These lubrication technology packages are pretested before delivery and consist of spray bars with individual nozzle-mixing units, an oil supply unit and a process unit with a defined interface to the mill automation system.

This paper deals with two innovative technology packages of Primetals for highly efficient and robust roll-gap lubrication solutions for hot rolling as well as cold rolling, which can be simply adapted to existing mills. The lubrication packages are characterized by a short ROI, which allow fully exploiting and extending the plant capability while reducing OPEX and adding value to your rolling products.

\section{MINIMUM QUANTITY LUBRICATION MQL® FOR COLD MILLS}

As one example of an innovative lubrication solution, Primetals Technologies has developed Minimum Quality Lubrication MQL®, which represents a new generation of advanced roll-gap lubrication systems for cold rolling mills. MQL $®$ applies pure rolling oil, finely atomized with air, directly onto the work roll surfaces. This allows for an intelligent control of the oil film thickness depending on the actual process and product parameters. In this way, MQL $®$ overcomes existing limitations of classical emulsion technologies in terms of flexibility (rapid change of highly different lubrication requirements) and ensures the adjustment of an optimum and productspecific lubrication level. Compared to conventional roll-gap lubrication with emulsion (either recirculation or direct application type), where the oil concentration inside the roll-bite mainly results from plate-out and wash-off effects, MQL® maximizes the level of oil concentration in the bite and therefore optimizes the lubrication performance and efficiency [2].

Figure 1 shows that with MQL® the entry side emulsion application is completely replaced by neat oil with very low flow rates through the top and bottom MQL $®$ oil/air mixing nozzles. The generated oil film layer on work roll surface can be precisely controlled and the amount of oil supplied to the roll bite can be adjusted in a flexible and efficient way. The main benefits of MQL $®$ are energy savings through reduced rolling forces and rolling torques, improved strip surface cleanliness and extended work roll lifetime (less strip wear and abrasion in the first mill stands, where MQL $®$ is 
preferably installed) and extended reduction capability (product mix extension). The total coolant/lubricant flow per mill stand is reduced, however, it could be shown that strip and work roll temperatures are only marginally increased provided that the exit side work roll cooling is working efficiently.

\section{Conventional emulsion lubrication}

MQL Iubrication

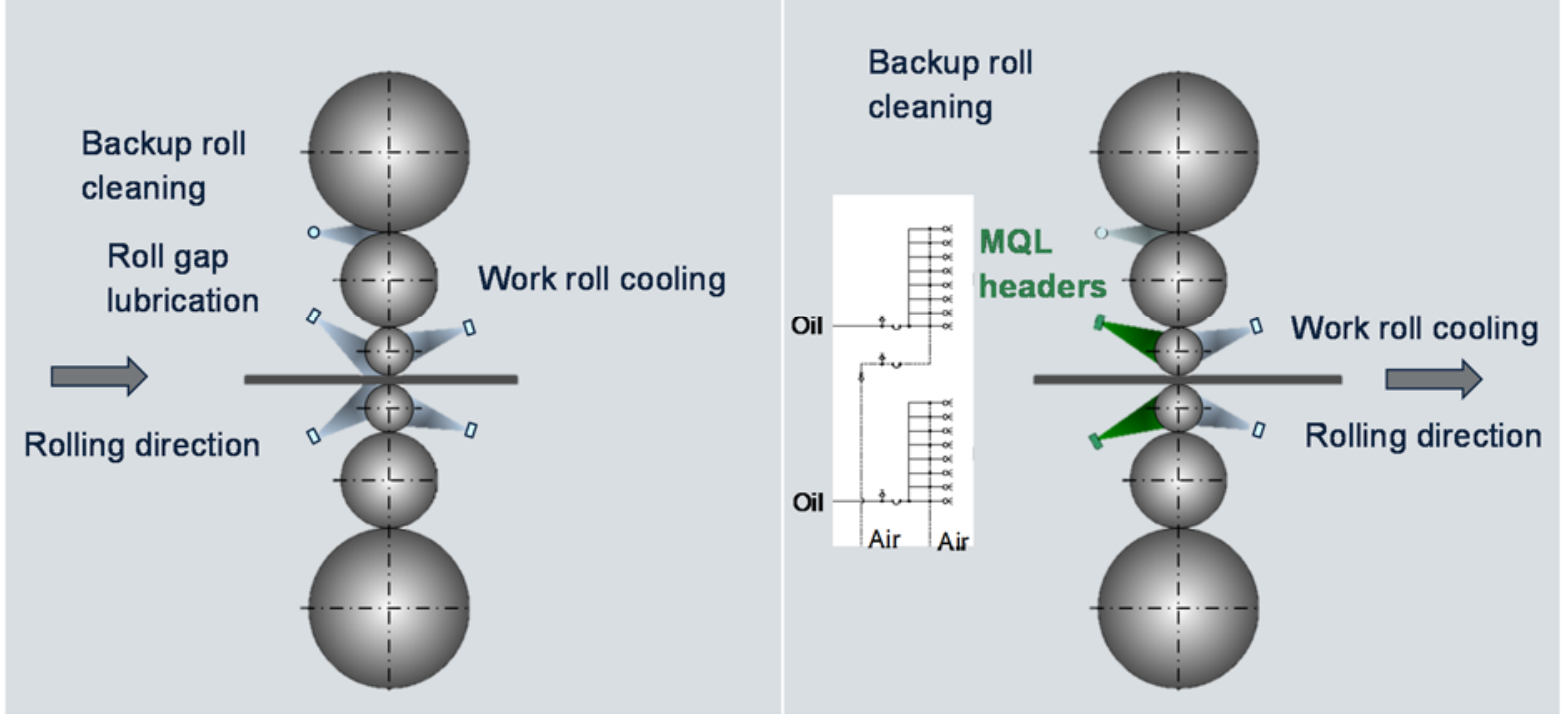

Figure 1. Typical MQL $®$ installation, oil/air schematic of spray headers.

\subsection{MQL® Prototype installation and test results on tandem cold mills}

MQL $®$ was successfully installed and tested on two different tandem cold rolling facilities in Europe: a coupled pickling line tandem cold mill (PLTCM) and a batch tandem cold mill. Figure 2 and 3 show the potential to decrease the rolling force in stand 1 of a PLTCM, where the strip enters the mill with pickling roughness and with dry surface. The friction conditions in the bite are immediately improved when the entry side emulsion flow is switched off and MQL® takes over (cf. Figure 2). The total roll force for this product decreases by about $14 \%$ until the end of the coil. An average roll force reduction of $8 \%$ could be achieved with MQL® in a batch of 21 identical coils compared to the average roll force with emulsion lubrication. For every single coil, the roll force level was below that of emulsion lubrication. All coils were

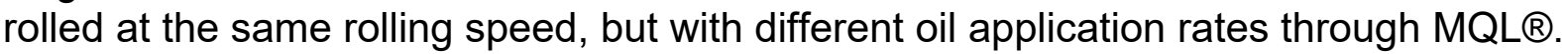
Rolling forces are equal between emulsion lubrication and $M Q L \circledR$ at a rate of approximately $400 \mathrm{mg} / \mathrm{m}^{2} /$ side. Below that level, the oil consumption with $\mathrm{MQL} \circledast$ is minimized at the cost of slightly increased rolling forces compared to those obtained with emulsion lubrication. Due to the fact that the oil is sprayed directly onto the work roll surface sufficiently before the contact zone, the restriction of low plate-out particularly observed with dry strip in the first stand (leading to poor film formation and high coefficients of friction), is overcome. The resulting roll force decrease may, if desired, be converted into higher reduction capabilities. MQL $®$ allows to adapt the lubrication strategies quickly and easily even from one product to the next. 


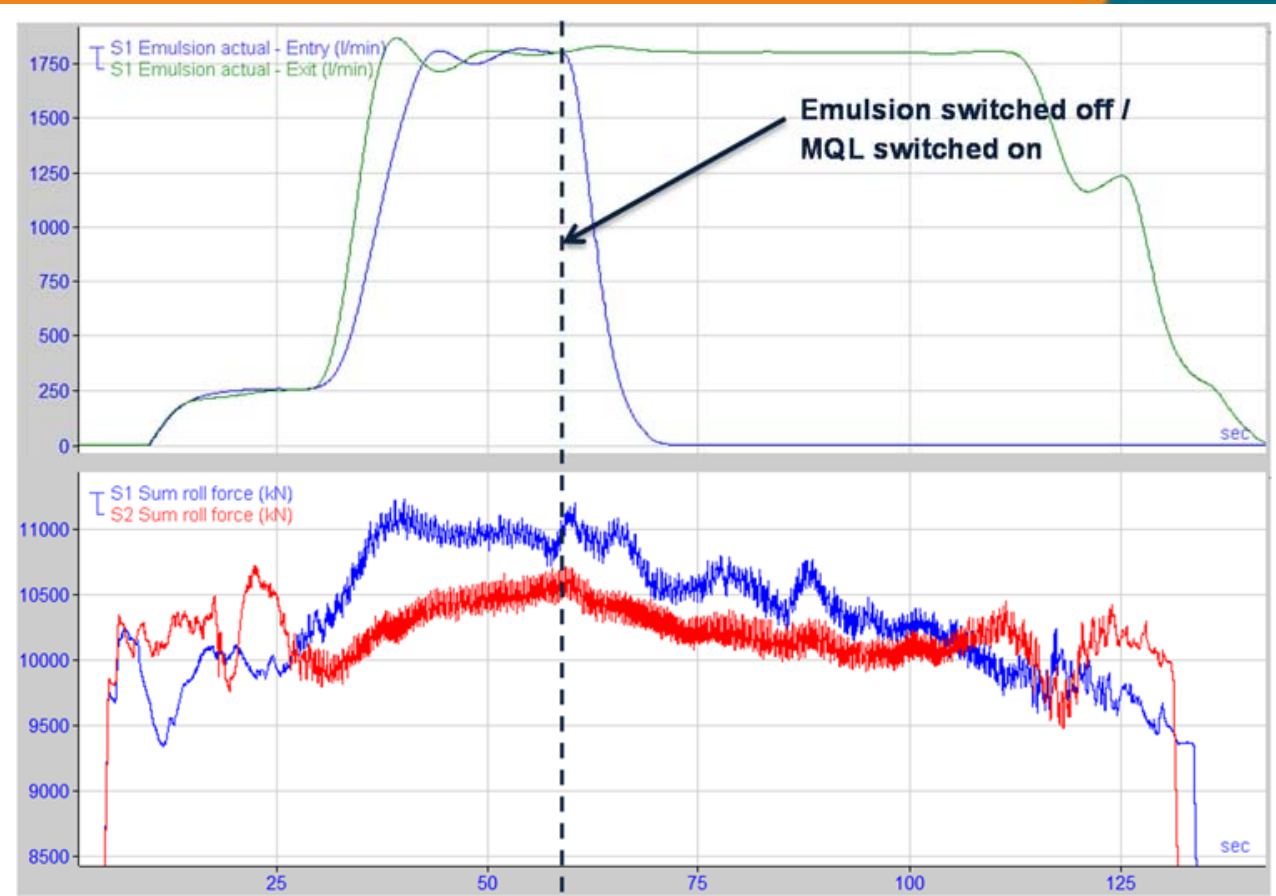

Figure 2. Rolling force reduction when emulsion lubrication is switched off during rolling (coil was finished with MQL® only).

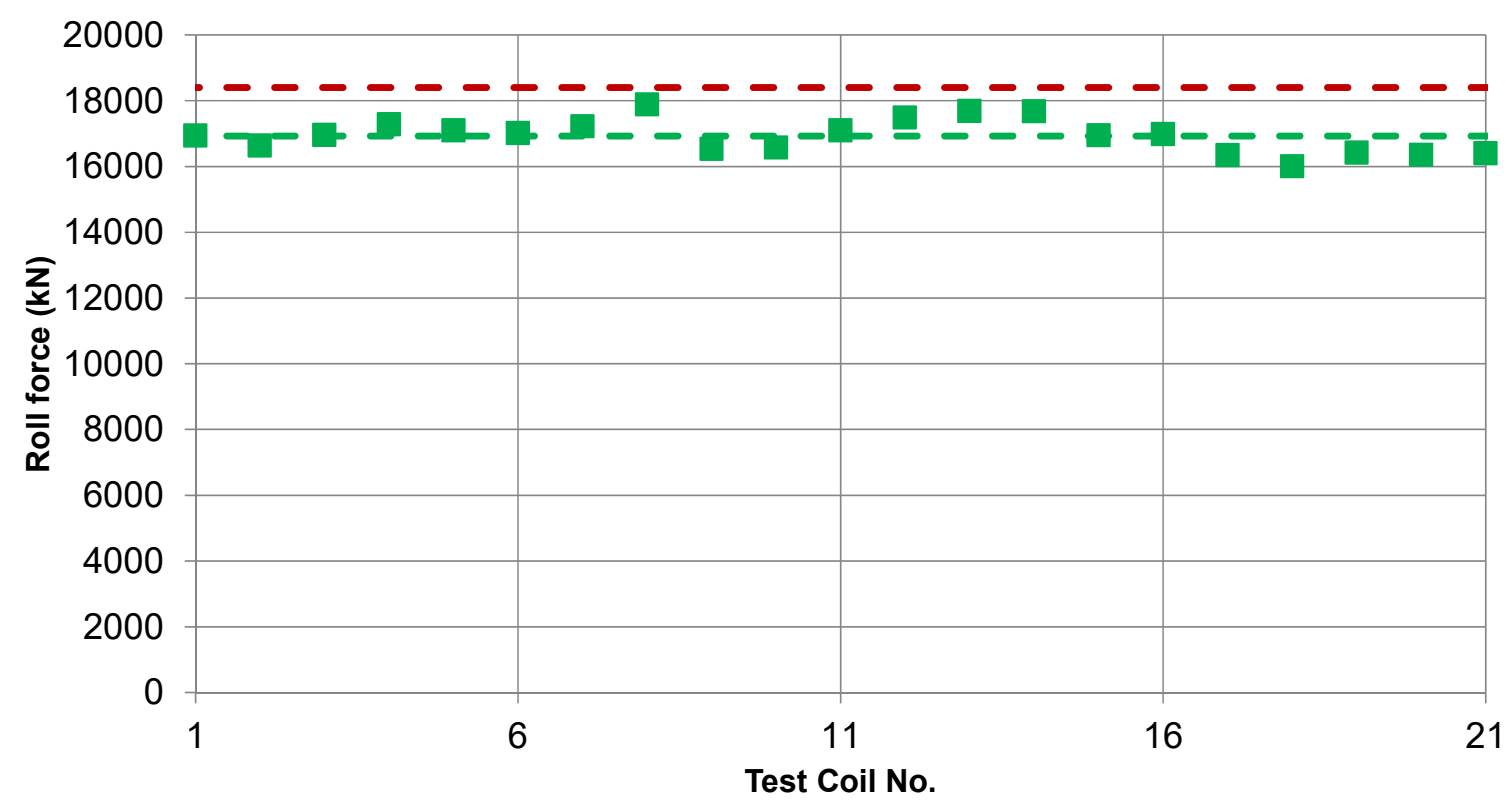

n Coils rolled with MQL - -Average roll force with emulsion - -Average roll force with MQL

Figure 3. Rolling force reduction with $\mathrm{MQL} \circledast$ compared to emulsion lubrication.

In a different campaign of 36 coils on this PLTCM, strip surface cleanliness was evaluated when rolling with MQL $®$ and emulsion lubrication (cf. Figure 4). Through improved lubrication conditions between the work roll and the strip, the amount of wear in stand 1 is reduced, which leads to a significant improvement in the strip surface reflectivity test (Scotch Tape Test) of around 10\% points on average. 


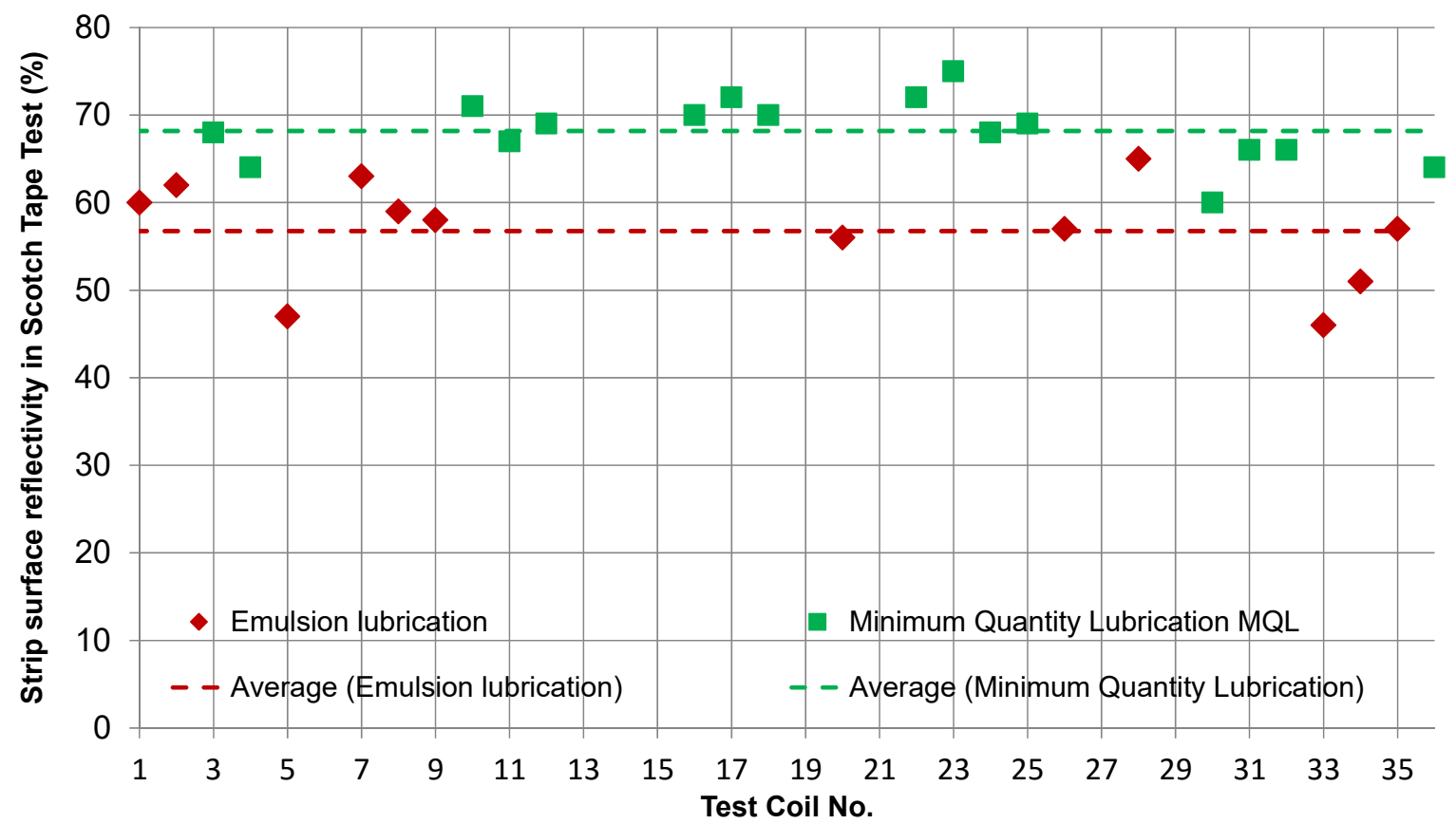

Figure 4. Strip surface cleanliness improvement with MQL® compared to emulsion lubrication.

On a batch tandem cold mill, rolling force reductions and strip surface cleanliness improvements of the same order of magnitude were observed for pre-oiled and dry incoming strip surface condition. Thanks to MQL® in the first stand it was furthermore possible to roll special products, which required pre-oiling when rolled with emulsion, in dry surface condition.

\section{ADVANCED WORK ROLL LUBRICATION A-WRL}

Primetals' advanced work roll lubrication (A-WRL) technology package for hot rolling and ESP plants stands meanwhile for a well proven, robust, reliable and eco-friendly solution with short return on investment (ROI).

To reach this goal, Primetals consequently puts effort in the development and optimization of all core components that are needed for the operational success of the system:

The "mixer-nozzle-unit", in which the water-oil-emulsion is mixed and then sprayed onto the work rolls through the nozzles, represents one of these core components. It is of vital importance to have a unit with minimum maintenance requirements and high availability in terms of nozzle clogging or contamination. On the other hand, the mixing quality has to be high and the spray pattern of the nozzles has to be as equal and uniform as possible to ensure that a uniform oil film is applied onto the work roll surface. Different space availability and requirements also made it necessary to develop specific mixer-nozzle-unit designs capable for this different requirement.

In addition to an optimized mixer-nozzle-unit, the used media (water and lubrication oil), which is mixed has to have specific properties to avoid nozzle clogging problems on the one hand, and to achieve a high plate-out effect on the work rolls and thus a high lubrication efficiency on the other hand.

Furthermore, the wiper material respectively the wiper tightness and its wear resistance play a decisive role. When a wiper becomes not watertight anymore, at least parts of the sprayed oil-water emulsion are washed away by cooling water and do not reach the work roll surface anymore. This results in lower lubrication 
efficiency, and can even lead to unsymmetrical lubrication over width in case that water tightness gets lost unequally over the wiper's width.

To be able to extensively test the behavior of new or redeveloped components inhouse, Primetals has set up a test-rig (cf. Figure 5) especially, for testing the efficiency of mixer-nozzle-unit configurations, as well as different lubrication media. Especially, the plate-out efficiency can be measured and the influence of parameter variations like spray angle, nozzle size, media amounts, oil type, oil temperature etc. can be studied in an easy and efficient way.

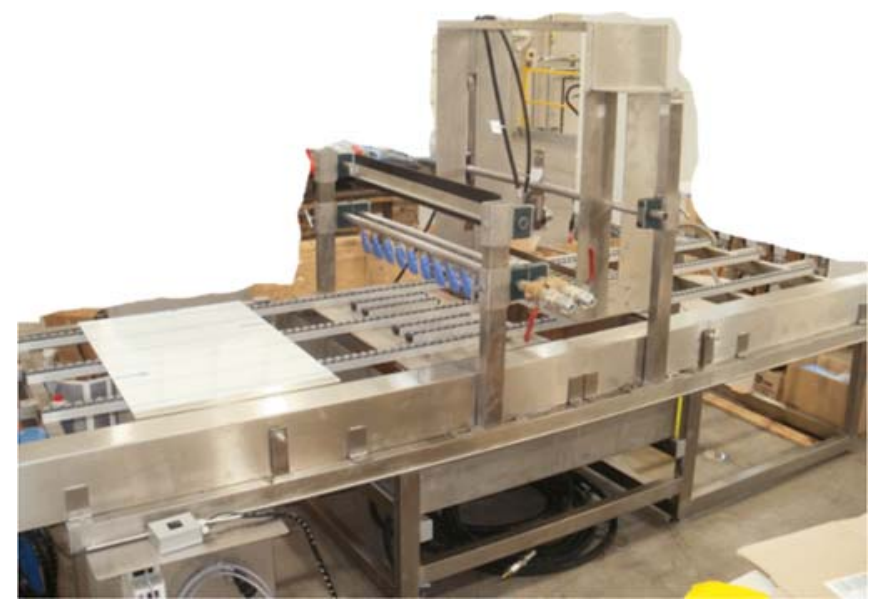

Figure 5. Test-rig for optimization of lubrication equipment core components.

\subsection{Mixer-nozzle-unit}

The advanced work roll lubrication package (A-WRL) for hot rolling and ESP plants is using specially designed mixer-nozzle units to mix the water/oil emulsion very close to the nozzles. This means that nozzle and mixer form a unit and are not separated from each other by interconnecting piping. With such a design, the risk for contamination and clogging of the nozzle unit is minimized, as the mixed emulsion is more or less immediately sprayed through the nozzle.

The mixer-nozzle units are designed to be quickly exchangeable and are optimized to ensure uniform lubricant distribution, high plate-out efficiency and low oil consumption, ensuring high roll force reduction and a significant decrease of work roll wear.
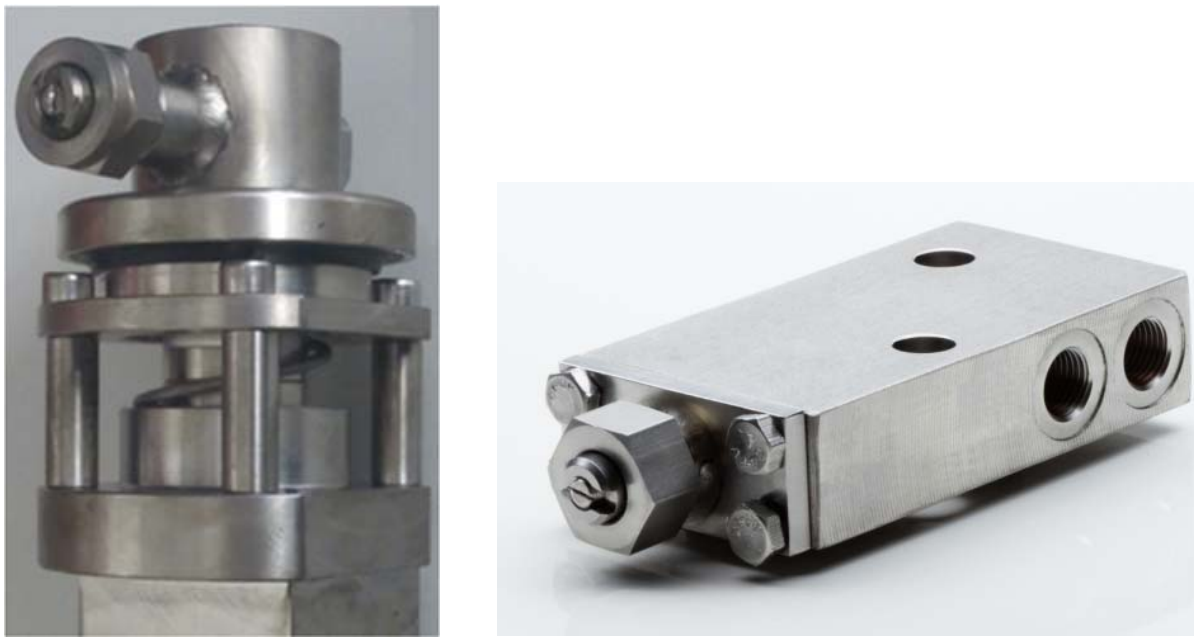

Figure 6. Advanced WRL mixer-nozzle unit designs for different space requirements. 
The mixer-nozzle-units are mounted at the wiper arm on opposite side to the entryside roll cooling system. This means that the roll cooling system can always be utilized without disturbing the lubrication efficiency. Both, the switched on cooling system, as well as the switched on lubrication system are of vital importance especially for high performance hot strip mills.

As an alternative, also a design where the mixer-nozzle-units, as well as the roll cooling system are located at the same side of the wiper can be the best solution depending on the requirements. See the information below on the product variant "WRL NC" for details.

Figure 7 shows a typical arrangement of the mixer-nozzle-units on the wiper arm:

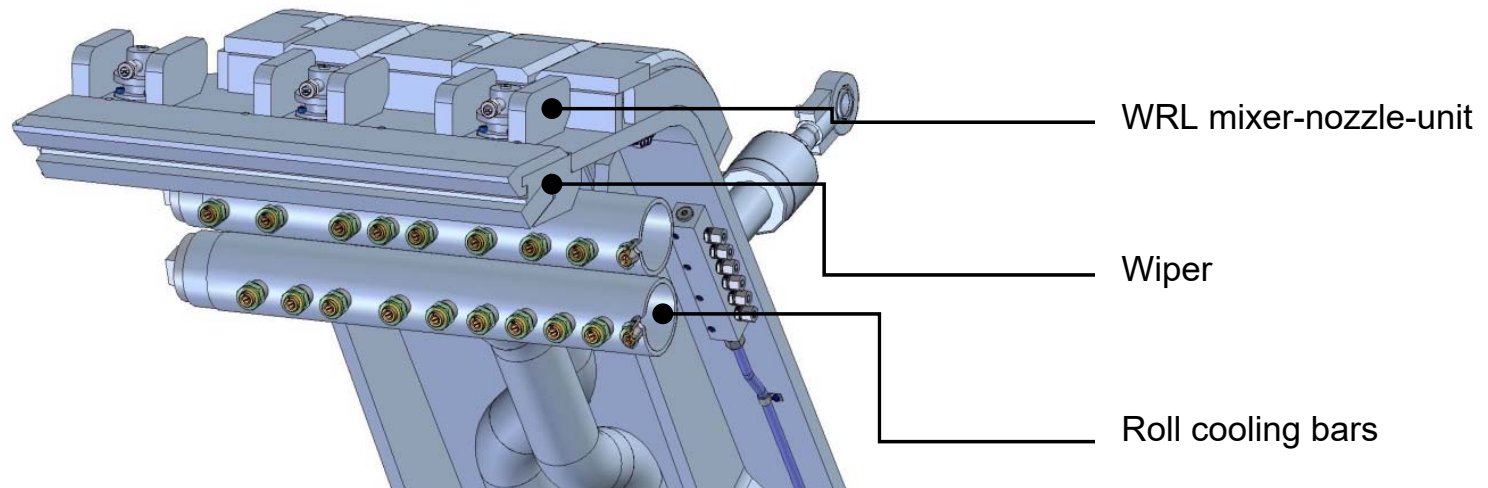

Figure 7. Typical arrangement of mixer-nozzle units on the wiper arm opposite to the roll cooling system.

\subsection{Wiper design and material}

The design of the wiper lip and the wiper material in combination with a proper pressing force of the wiper arm is a key prerequisite for achieving a uniform and efficient lubrication result. Therefore, Primetals tested different kind of wiper designs and materials to finally choose the design and material which best fits to the requirements.

\subsection{An eco-friendly solution}

As the water-oil emulsion is mixed directly in front of the nozzle, it is not necessary to use any chemical additives or cleaning agents to regularly free pipes from contamination.

Depending on the width of the actual rolled strip, the outermost nozzles are automatically activated or deactivated in order to avoid spraying useless oil outside of the actual rolled dimension.

Depending on the rolled material and dimensions, typical roll force reductions of 10$20 \%$ are adjusted at commissioning by optimizing the amount of lubrication oil for different rolled materials. This subsequently results in a significant decrease of the consumed main drive power.

Due to the additional lubrication media brought into the roll gap, the friction coefficient between work roll surface and strip surface is lowered, which results in a significant decrease of work roll wear on the one hand, and an increased strip surface quality on the other hand [3].

Figure 8 shows a typical case where the lubrication system is activated shortly after the respective mill stand is loaded. The roll force is decreased as long as the 
lubrication is switched on and immediately increases when the system is switched off shortly before thread-out of the strip.

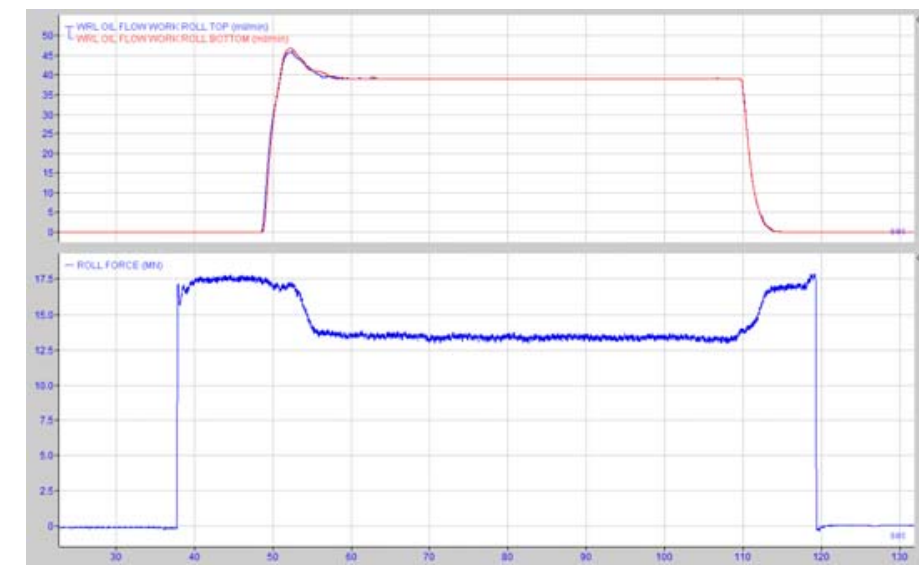

Amount of lubrication oil

Roll force

Figure 8. Example for typical roll force reduction with activated advanced WRL system.

\subsection{Product variant WRL NC (Work roll lubrication "non cooling")}

In a cooperation project with a leading steel producer, a new variant for a WRL system has been jointly designed for the given requirements and the package was piloted at a conventional hot strip mill.

Major difference to the advanced WRL concept is that the mixer-nozzle-unit and the roll cooling system are located at the same side of the wiper arm.

An advantage of this design is that the mixer-nozzle-unit is physically separated from the hot strip by the wiper mechanics, which, of course, has positive influence on the robustness of the system e.g. in case of cobbles. On the other hand, the efficiency of this design comes to its limitations when lubrication as well as roll cooling is switched on at the same time. In such a situation, only a minor or even no lubrication effect is visible anymore, as the roll cooling water washes off parts of the oil before it can be transported into the roll gap.

Therefore, such a design especially makes sense for plants/materials where the entry side roll cooling can be switched off as long as the mill stand is loaded or at least as long as lubrication is switched on.

Considering the advantages and disadvantages of these two designs in combination with the mill roll cooling capabilities, it has to be decided case by case, which design best fits to a given plant configuration.

\subsection{Outlook on new and future developments}

Present development activities are focused on enhancing the work roll lubrication system for a safe continuous lubrication operation from the very beginning of the strip until the end of the strip. Therefore, at least for limiting strips in terms of the bite angle and the friction coefficient, possible remaining lubrication oil has to be safely removed from the work roll surface before thread in of the next strip.

Further, core components are continuously optimized to guarantee that the Primetals advanced work roll lubrication system adds value to our customers' products. 


\section{CONCLUSION}

The presented paper focuses on two innovative technologies for highly efficient rollgap lubrication for hot and cold rolling, which were developed utilizing extensive knowledge and expertise about the tribological interactions in the roll-gap.

Advanced WRL and MQL represent Primetals' latest technologies that feature robust and reliable systems comprising standardized interfaces and flexible communication options. Both lubrication packages are optimized, pre-tested before delivery and can easily be integrated into existing or new hot strip mills, ESP (Endless Strip Processing) lines or cold rolling mills.

Product-specific lubrication contributes to energy savings and reduction of roll wear in standard product mixes or helps to extend plant capability towards more challenging products - with an improvement in product quality in both cases.

\section{REFERENCES}

$1 \quad$ M. Bergmann, A. Kainz, K. Zeman, K. Krimpelstaetter, D. Paesold, B. Smeulders and P. Schellingerhout, Enhanced Modelling of Friction and Lubrication in Cold Strip Rolling, Proceedings of the $9^{\text {th }}$ International $\& 6^{\text {th }}$ European ROLLING Conference (2013)

2 J.A. Schey, Tribology in Metalworking: Friction, Lubrication and Wear, ASM Intl., (1983)

3 T. Braidt, A new generation of Roll Gap Lubrication for Hot Strip Mills, AISTech Conference Proceedings (2013) 